\title{
ON THE PERIODICITY OF HUBBLE-SANDAGE OBJECTS IN M 31
}

\author{
G. R. IVANov \\ Department of Astronomy, University of Sofia, 1126 Sofia, Bulgaria
}

The spectral and photometric characteristics of the Hubble-Sandage objects (HSO) are similar to those of the $\eta$ Car and S Dor variables. They are actually referred to as S Dor-type in the General Catalogue of Variable Stars. Their light variations differ from star to star.

The aim of the present paper is to discuss a possible periodicity in HSOs in M 31 . We used phtometry from Hubble(1929), Sharov(1973, 1981), Rosino and Bianchini(1973), and added some observations taken during 1980-87. The latter were obtained with the 2-m Ritchey-Chretien-Coude and 0.5-m Schmidt telescopes of the Bulgarian National Astronomical Observatory - Rozhen.

Table 1 containes the observational data on four HSOs. Columns 2-4 contain data from nine Schmidt-telescope plates. The absorption in the line of sight in the Galaxy is adopted to be $E_{B-V}=0.08$ and within the M31 associations it is up to 0.24 (Massey et al.,1986). Hence we decided to use $E_{B-V}=0.08$ for $A E$ And and $A F$ And. The variables VA-1 and V15 are located within the spiral arms near dust clouds and they are probably influenced by internal absorption in M31. For both of them we used $E_{B-V}=0.24$. In luminosity the HSOs are similar to the brightest blue supergiants. Their location on the colour-magnitude diagram suggests that they are young massive stars in a rapid evolutionary stage.

The HSOs are irregular variables. Still we attempted to look for periodicity using the method of Deeming(1975) - Fourier analysis. The periodicities we find range from 800 to 3700 days (Table 1). And indeed, Hubble(1929) noted a five-year cycle in AF And, which coincides with our value. The period-luminosity diagram of the M31 Cepheids is shown in Fig. 1. The four HSOs are marked with crosses. It appears that they lay well on the continuation of the period-luminosity relation.

Back in the 50s, Hubble and Sandage(1953) suggested that HSOs are appropriate as extragalactic distance indicators. However, their 5 to 10 - year-long cycles make it inpractical for them to be used as such. Looking for possible explanations to Fig. 1, let us note that the equation $\mathrm{P} \sqrt{\rho}=\mathrm{Q}$ could be transformed to:

$\log \mathrm{P}+0.248 \mathrm{M}_{\text {bol }}+3 \log \mathrm{T}_{\text {eff }}-12.50=\log \mathrm{Q}$

We used the relations of deJager (1980) for converting the intrinsic colours $B-V$ and $M_{B}$ into $\mathrm{T}_{\text {eff }}$ and $\mathrm{M}_{\text {bol }}$. The data from Table 1 give $\mathrm{Q}=25.1$. This value is too large to explain the luminosity variations by pulsations.

The spectroscopic observations of Kenyon and Gallagher(1985) suggested that the HSOs might be massive binary systems. Variations in the properties of the stellar wind could be a possible cause for their variability. 
TABLE 1

HUBBLE-SANDAGE OBJECTS IN M 31

$\begin{array}{lllll}\text { STAR } & \text { B } & \text { B.V } & M_{B} & \text { PERIODICITY (days) }\end{array}$

$\begin{array}{lllll}\text { AF And } & 16.6 & -0.02 & -7.8 & 1700 \\ \text { AE And } & 17.1 & 0.0 \mathrm{i} & -7.3 & 1200 \\ \text { VA-1 } & 16.8 & 0.20 & -8.1 & 3670 \\ \text { V } 15 & 18.1 & 0.40 & -6.8 & 862\end{array}$

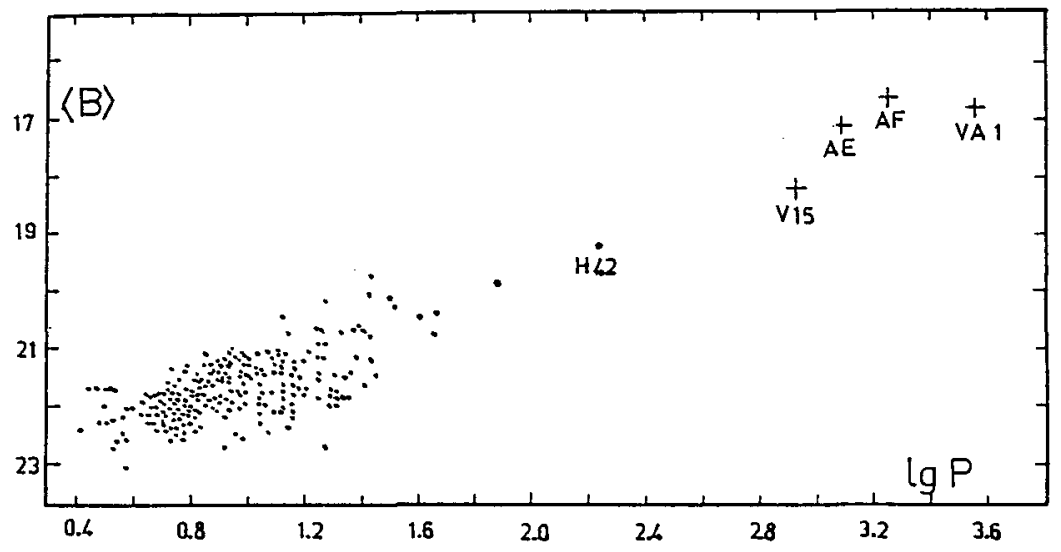

REFERENCES

Deeming, T.J. 1975, Ap. Space Sci., 36, 137.

De Jager, C. 1980, The Brightest Stars (D.Reide1: Dordrecht).

Hubble, E.P. 1929, Ap. J., 69, 103.

Hubble, E.P., and Sandage, A.R. 1953, Ap. J., 118, 353.

Kenyon, S.J., and Gallagher III,J.S. 1985, Ap. J., 290, 542.

Massey, P., Armandroff, T.E., and Conti, P.S. 1986, A.J., 92, 1303.

Rosino, L., and Bianchini, A. 1973, Astr. Ap., 22, 453.

Sharov, A.S. 1973, Peremenye Zvezdy, 19, 3.

Sharov, A.S. 1981, Peremenye Zvezdy, 21, 485. 